$(p=0.026 ; O R=0.16 ; 95 \% \mathrm{Cl}=[0.03-0.79]$ and $p=0.046 ; O R=0.2 ; 95 \%$ $\mathrm{Cl}=[0.04-1.00]$, while for $D R B 1^{*} 03: 01$ negative association was revealed only for systemic JIA $(p=0.03 ; O R=0.11 ; 95 \% \mathrm{Cl}=[0.01-0.88])$. At the same time the $D Q B 1^{*} 04: 02: 01 \quad(\mathrm{p}=0.026 ; \mathrm{OR}=5.88 ; 95 \% \mathrm{Cl}=[1.20-28.72])$ and $D R B 1 * 08: 01: 01(\mathrm{p}=0.07 ; \mathrm{OR}=3.94 ; 95 \% \mathrm{Cl}=[1.01-15.39])$ allele frequencies were significantly higher in patients with oligoarthritis but not systemic JIA when compared with controls.

Conclusion: High-throughput HLA typing revealed distinct HLA-alleles associated with different JIA subtypes in the Belarusian population as well as some alleles protective both for oligoartricular and systemic JIA.

Disclosure of Interests: None declared

DOI: 10.1136/annrheumdis-2021-eular.1346

\section{AB0004 THE ASSOCIATION OF THE RARE RS35667974 IFIH1 GENE POLYMORPHISM WITH SIX AUTOIMMUNE DISEASES: STRUCTURAL BIOLOGICAL INSIGHTS}

M. Zervou ${ }^{1}$, A. Andreou ${ }^{2}$, G. Goulielmos ${ }^{1}$, E. Eliopoulos ${ }^{2} .{ }^{1}$ University of Crete, Heraklion, Greece, Section of Molecular Pathology and Human Genetics, Department of Internal Medicine, School of Medicine, Heraklion, Greece; ${ }^{2}$ Agricultural University of Athens, Department of Biotechnology, Athens, Greece

Background: Genome wide association studies (GWAS) have successfully identified novel autoimmune disease-associated loci, with many of them shared by multiple disease-associated pathways but much of the genetics and pathophysiological mechanisms remain still obscure. Considering that most of the potential causal variants are still unknown, many studies showed that the missense variant rs35667974 at interferon induced with helicase $\mathrm{C}$ domain 1 (IFIH1) gene is protective for type 1 diabetes (T1D), psoriasis (PS) and psoriatic arthritis (PsA), while it was found to be also associated with ankylosing spondylitis (AS), Crohn's disease (CD) and ulcerative colitis (UC). IFIH1 gene encodes a cytoplasmic RNA helicase that recognizes viral RNA and is involved in innate immunity through recognition of viral RNA [1].

Objectives: To investigate the role of the rare rs35667974 variant of IFIH1 gene, which resides in exon 14 and changes a conserved isoleucine at position \#923 to valine in T1D, PS, PsA, AS, CD and UC [2-4] as well as the mechanism that may affect the function in the protein structure.

Methods: Evolutionary analysis revealed heavily conserved sequence elements among species, indicating structural/functional importance of the mutation at position \#923. In silico mutagenesis and three-dimensional (3D) homology modeling was used to localize the polymorphism under study on the IFIH1 protein. The mutant was constructed using molecular modeling with the program Maestro (Schrodinger, LLC) [5]. Molecular mechanics/dynamics studies were applied to validate structural/functional changes caused by the lle923V substitution. All figures depicting 3D models were generated using the PyMOL molecular-graphics system V.2.2 [6].

Results: Evolutionary and structural analysis revealed that the position of residue lle923 is located on a protein loop (921-927) directly interacting with mRNA both to the phosphoribose chain and the base pairs. Mutation of lle to Val at position \#923 will directly affect the said interaction with mRNA [7] (Figure 1). Moreover, it has been reported that pre-mRNA or mRNA levels did not correlate with Ile923Val, suggesting that, lle923Val did not alter the expression of IFIH1 in a major way [7].

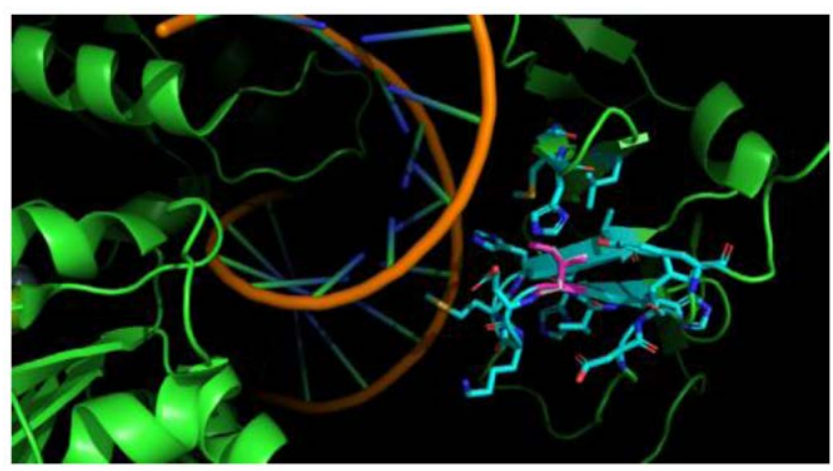

Figure 1. Structural representation of the interferon-induced helicase $C$ domain-containing protein 1 [Homo sapiens] structure (4GL2 from Protein Data Bank) (in green) bound to the double stranded RNA (in orange-yellow). The location of the mutation (I923V) is highlighted in pink. The proximity of the aminoacid 923 to the nucleotide is apparent.
Conclusion: This study represents a comprehensive evaluation of the role of the shared rs35667974 variant of autoimmune locus IFIH1, reported to lead to a loss of function phenotype and, as a consequence, reduced levels of IFIH1 protein and activity that protect against autoimmunity. Structural analysis of rare shared genetic susceptibility or protection loci may provide insight to our understanding of the pathophysiology of autoimmune diseases and the research findings may affect the better management of the diseases under study.

REFERENCES:

[1] Nejentsev S et al. (2009). Science 324:387-389.

[2] Smyth DJ et al. (2006). Nat Genet. 38: 617-619.

[3] LiY et al. (2010). J Invest Dermatol 130:2768-2772.

[4] Ellinghaus D et al. (2016). Nat Genet 48:510-518.

[5] Schrödinger Release 2017-1: Maestro, Schrödinger, LLC, New York, NY 2017.

[6] Schrödinger LLC: The PyMOL Molecular Graphics System 2016 version 2.2 .

[7] Wu B et al. (2013). Cell 152:276-289[8] Downes K et al. (2010). PLoS One 5:e12646.

Disclosure of Interests: None declared

DOI: 10.1136/annrheumdis-2021-eular.1388

\section{AB0005 $\quad$ IDENTIFICATION OF KEY GENES AND PATHWAYS FOR PSORIASIS BASED ON GEO DATABASES BY BIOINFORMATICS ANALYSIS}

X. Sun ${ }^{1}$, S. X. Zhang ${ }^{2,3,4}$, S. Song ${ }^{2,3,4}$, T. Kong ${ }^{1}$, C. Zheng ${ }^{1}$, L. Cheng ${ }^{1}$, S. Feng ${ }^{5}$ G. Shi ${ }^{6}$, X. LI ${ }^{2,3,4}$, P. F. He ${ }^{7}$, Q. Yu ${ }^{1,7}$. 'Shanxi Medical University, School of Management, Taiyuan, China; ${ }^{2}$ The Second Hospital of Shanxi Medical University, Department of Rheumatology, Taiyuan, China; ${ }^{3}$ Shanxi Li Xiaofeng Medical Groups, Department of Rheumatology, Taiyuan, China; ${ }^{4}$ Ministry of Education, Key Laboratory of Cellular Physiology at Shanxi Medical University, Taiyuan, China; ${ }^{5}$ Shanxi Medical University, School of Humanities and Social Science, Taiyuan, China; ${ }^{6}$ Shanxi Medical University, Basic Medical College Taiyuan, China; ${ }^{7}$ Shanxi Medical University, Institute of Medical Data Sciences, Taiyuan, China

Background: Psoriasis is an immune-mediated, genetic disease manifesting in the skin or joints or both, and also has a strong genetic predisposition and autoimmune pathogenic traits ${ }^{1}$. The hallmark of psoriasis is sustained inflammation that leads to uncontrolled keratinocyte proliferation and dysfunctional differentiation. And it's also a chronic relapsing disease, which often necessitates a longterm therapy ${ }^{2}$.

Objectives: To investigate the molecular mechanisms of psoriasis and find the potential gene targets for diagnosis and treating psoriasis.

Methods: Total 334 gene expression data of patients with psoriasis research (GSE13355 GSE14905 and GSE30999) were obtained from the Gene Expres sion Omnibus database. After data preprocessing and screening of differentially expressed genes (DEGs) by $R$ software. Online toll Metascape ${ }^{3}$ was used to analyze Gene Ontology (GO) and Kyoto Encyclopedia of Genes and Genomes (KEGG) pathway enrichment analysis of DEGs. Interactions of proteins encoded by DEGs were discovered by Protein-protein interaction network (PPI) using STRING online software. Cytoscape software was utilized to visualize PPI and the degree of each DEGs was obtained by analyzing the topological structure of the PPI network

Results: A total of 611 DEGs were found to be differentially expressed in psoriasis. GO analysis revealed that up-regulated DEGs were mostly associated with defense and response to external stimulus while down-regulated DEGs were mostly associated with metabolism and synthesis of lipids. KEGG enrichment analysis suggested they were mainly enriched in IL-17 signaling, Toll-like receptor signaling and PPAR signaling pathways, Cytokine-cytokine receptor interaction and lipid metabolism. In addition, top 9 key genes (CXCL10, OASL, IFIT1, IFIT3, RSAD2, MX1, OAS1, IFI44 and OAS2) were identified through Cytoscape.

Conclusion: DEGs of psoriasis may play an essential role in disease development and may be potential pathogeneses of psoriasis.

\section{REFERENCES:}

[1] Boehncke WH, Schon MP. Psoriasis. Lancet 2015;386(9997):983-94. doi: 10.1016/S0140-6736(14)61909-7 [published Online First: 2015/05/31].

[2] Zhang YJ, Sun YZ, Gao XH, et al. Integrated bioinformatic analysis of differentially expressed genes and signaling pathways in plaque psoriasis. Mol Med Rep 2019;20(1):225-35. doi: 10.3892/mmr.2019.10241 [published Online First: 2019/05/23]

[3] Zhou Y, Zhou B, Pache L, et al. Metascape provides a biologist-oriented resource for the analysis of systems-level datasets. Nat Commun 2019;10(1):1523. doi: 10.1038/s41467-019-09234-6 [published Online First 2019/04/05]. 